\title{
Challenges in recruitment of research participants
}

\author{
Maxine X. Patel, Victor Doku \& Lakshika Tennakoon
}

\begin{abstract}
There are many potential pitfalls in the identification and enlistment of suitable candidates for psychiatric research. The challenges of recruitment are highlighted, detailing impact of study design, characteristics of participants, including demographics and personal preferences, investigator characteristics and collaboration with clinicians. Techniques used in recruitment are discussed, including financial incentives, assertive tracking and communication methods. Ethical issues, methods of data collection, and control participants are also considered. Key issues are: early consideration of the impact of study design on the recruitment process; the participant's perspective; close collaboration with colleagues; the investigator's good interpersonal, communication and organisational skills; and feedback to collaborators, associated clinicians and participants.
\end{abstract}

Recruitment is the dialogue which takes place between an investigator and a potential participant prior to the initiation of the consent process. It begins with the identification, targeting and enlistment of participants (volunteer patients or controls) for a research study. It involves providing information to the potential participants and generating their interest in the proposed study. There are two main goals of recruitment:

- to recruit a sample that adequately represents the target population;

- to recruit sufficient participants to meet the sample size and power requirements of the study (Hulley et al, 2001; Keith, 2001).

Problems with recruitment can disrupt the timetable for a research project, preoccupy staff, reduce the ability of a therapeutic study to detect treatment differences and, ultimately, result in a trial being abandoned (Ashery \& McAuliffe, 1992).

During recruitment, the sampling process can suffer from associated problems of non-response and the resultant selection bias. The proportion of eligible participants who agree to enter the study (the response rate) influences the validity of the inference that the sample represents the population of interest (Woods et al, 2000; Hulley et al, 2001). People who are difficult to reach and those who refuse to participate once they have been contacted tend to be different from people who do not enrol. A summary of participant characteristics often associated with poor response is provided in Box 1 (see Armstrong et al, 1992).

Retention of participants is a related and important aspect of recruitment. Poor retention is costly

\section{Box 1 Main factors adversely affecting response rates}

\author{
Greater age \\ Male gender \\ Non-White race \\ Urban residence \\ Low educational status \\ Unemployed or low occupational status \\ Low family income \\ Smoker \\ Recent illness or poor present health \\ High use of medical care
}

\footnotetext{
Maxine Patel is a Medical Research Council Special Training Fellow (Health Services Research) at the Institute of Psychiatry and GKT School of Medicine (Division of Psychological Medicine, 103 Denmark Hill, London SE5 8AZ, UK. E-mail: m.patel@ iop.kcl.ac.uk) and an honorary specialist registrar in psychiatry. Her research interests include patient and staff attitudes to medication used in psychoses, as well as insight and treatment adherence in psychoses, mood disorders and depersonalisation. She has received funding through an investigator-initiated grant from Janssen-Cilag and consultation fees from the pharmaceutical industry. Victor Doku is a Wellcome Trust Research Fellow in Tropical Medicine and an honorary specialist registrar in psychiatry at the Institute of Psychiatry. His research interests include the epidemiology of psychoses in Africans, ethnopsychopharmacology of Africans and pragmatic clinical trials in developing countries. Victor Doku has worked as a subinvestigator on international trials on first-episode psychosis sponsored by Eli Lilly and Janssen-Cilag. Lakshika Tennakoon is a research scientist at the Mental Health Centre of Boulder County, Boulder CA, USA. She has previously worked at the Institute of Psychiatry, London, and the London School of Hygiene and Tropical Medicine. Her research interests include family caregivers in schizophrenia and cancer populations and epidemiological aspects of schizophrenia and other chronic disorders.
} 
both financially and in terms of time. Early retention techniques should be incorporated into recruitment strategies during the planning phase of the study (Nishimoto, 1998). Retention also involves building relationships with participants to encourage their continuing participation.

Recruitment is perhaps the most challenging part of a clinical research study. It is often left for junior and inexperienced researchers to undertake and it is not often reported adequately in the literature (Dowling \& Wiener, 1997). Participant recruitment is also dependent on the type of study undertaken, collaboration with the clinician, characteristics and preferences of the participants and the recruitment strategies employed. The aim of this article is to inform clinicians about these key issues for research on clinical diseases and treatment methods in psychiatry.

\section{Design issues}

Factors that need to be taken into consideration when recruiting participants include: sample size requirements; ethical principles; the type of study design; and the data collection methods (questionnaire, interview techniques) to be used. The main types of study design are shown in Fig. 1.

\section{Sample size and ethical principles}

Estimating the required sample size is one of the most important aspects of the recruitment process. Before a study is designed, it is important (if not mandatory) to calculate the sample size necessary to show a clinically important result. A sample size too small to detect differences could lead to studies which are unethical or wasteful. Furthermore, it can produce misleading results. For example, the results may show that there is no difference between groups or association between variables, when in fact there is a difference or an association (a Type II error). In contrast, a sample size that is too large leads to unnecessary expenditure of time, effort and finance.
Box 2 Content of participant information sheet and consent form

Names of the investigators

Purpose of the research

Description of procedures

Estimate of time required

Potential benefits and risks of the procedures

Procedures to maintain confidentiality / anonymity

A statement that potential participants can refuse to participate or withdraw from the study at any time without prejudicing their care/interests (the right to refuse/withdraw)

An explicit offer to answer questions or provide further information, and whom to contact

To minimise the probability of such errors, a power calculation is usually undertaken at the design stage. (Power can be defined as the probability of correctly concluding that there is a difference, given the sample size.) For these reasons researchers must make allowances in terms of time and money for the recruitment of a desired sample size (Streiner, 1990; Hulley et al, 2001).

As ethical principles must be adhered to and allowance made for potential participants who decline to take part, the required sample size needs to be adjusted to reflect the refusal rate. Ethical principles are based on the respect of the researcher for all potential participants and thus require the researcher to (i) obtain informed consent, (ii) protect participants with impaired decision-making capacity and (iii) maintain confidentiality (Hulley et al, 2001). Informed consent is the process by which a fully informed individual voluntarily decides whether or not to take part as a research participant. The discussion between the recruiting researcher and potential participant should include the following: the nature of the procedure; reasonable alternatives to any proposed intervention; the relevant risk; benefits and uncertainties relating to each alternative; assessment of the potential

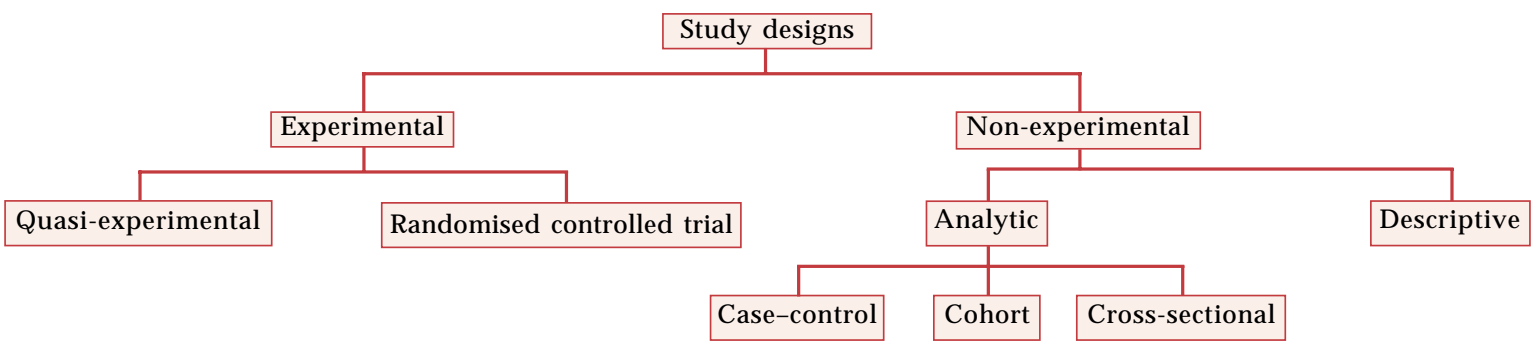

Fig. 1 Classification of study designs. 
participant's understanding; and the participant's acceptance of the intervention (see Box 2). The language used in the process should be pitched at a level that can be understood by the potential participants. For informed consent to be valid, the person concerned must be competent to make a decision and the consent must be voluntary (Beauchamp et al, 1991; Carpenter, 1999; Michels, 1999). To avoid any suggestion of coercion, which can easily occur in clinical situations, adequate time must be allowed for the patient to comprehend and give feedback (i.e. between the initiation and completion of the consent process). An information sheet and a consent form are required and the latter is signed by the participant and the researcher at the end of process.

\section{Cross-sectional design}

In a cross-sectional study, all the measurements of risk factors, outcome and confounding variables are made at a single point in time or over a short, specified period with no requirement for a followup period. For example, cross-sectional studies can provide data on the disease prevalence and distribution of the aforementioned variables. Recruitment for a cross-sectional study is generally less difficult than that for cohort studies and randomised controlled trials, because it involves one-off data collection with no commitment to follow-up.

\section{Case-control design}

In a case-control study, individuals with a particular condition or disease (the cases) are compared with a series of individuals in whom the condition is absent (the controls). Retrospective comparison between the two groups can then show differences in predictor variables (using assessments / measurements) that might explain why the cases developed the disease but the controls did not. Case-control studies are susceptible to a sampling bias by the recruiting researchers. Separate sampling of cases and controls, and the retrospective measurement of predictor variables, can bias the results and the conclusions drawn from them. This can be minimised by careful attention to the recruitment of a representative sample of cases and selection of appropriate controls.

\section{Cohort studies and randomised controlled trials}

In a cohort study, individuals are identified and grouped according to whether or not they possess a risk factor that is of interest (prospectively). The participants are then followed up over time and the incidence of the outcome (disease) is compared between the two groups (according to their risk factor status).

In a randomised controlled trial, participants are randomly allocated to one of two or more different groups, which are then treated in different ways (prospectively). The groups are followed up over time and measures of outcome (disease) are compared at the end of the study.

It is difficult to recruit participants for these studies because they often require a commitment to long-term follow-up. Both types of study usually require large sample sizes. Difficulties may arise with recruitment for cohort studies when participants do not see the point of the research because they have not yet developed the disease being studied. Recruitment for a randomised controlled trial is usually more difficult than that for an observational study because the participants must be willing to be assigned randomly and to take or accept treatments to which they may have been blinded. The possibility of receiving a placebo treatment is often a source of concern (Hulley et al, 2001).

\section{Data collection}

The two most common methods of data collection in psychiatric clinical research are questionnaires and interviews. Questionnaires are usually selfreport instruments, whereas interviews are administered verbally by the researcher. Each approach has costs and benefits and implications, therefore, for participant recruitment. Questionnaires are commonly used with cross-sectional survey designs. They are generally a more efficient, uniform and cheaper way to administer simple questions. It is usually better to use interviews to collect answers to complicated questions that require explanation or guidance. Research interviewers can ensure that the instruments are answered completely, probing when the responses being volunteered fall short of what is required. Interviews may be necessary when participants have different levels of reading ability and might find it difficult to understand the questions. However, interviews are more costly and time-consuming and the responses may be influenced by the relationship between the interviewer and the respondent (Polgar \& Thomas, 2000; Hulley et al, 2001). For example, interviewers can increase the magnitude of the error if, by their appearance, manner, method of administration of the interview or method of recording of the responses, they exert a qualitative influence on participants' responses (Armstrong et al, 1992). Newer and alternative methods of data collection include computer-assisted telephone interviewing 
and internet-based (online) questionnaires (Smith \& Senior, 2001).

The choice of data collection method is often dictated by practical considerations such as costs and logistics. When two methods are feasible, the choice involves trade-offs between cost-effectiveness, complexity of the questions to be answered and the required response rate. Sometimes a combination of administration methods is used. Within a single assessment, the number of instruments used should be minimised to prevent excessive participant burden. With repeat assessments over a period of time (i.e. follow-up studies), use of numerous instruments will increase investigator burden and will also contribute to poor recruitment. A cardinal rule is that it is usually not necessary to administer all instruments at every assessment.

\section{Choosing the participant group}

Before beginning the recruitment process, it is critical that the essential inclusion and exclusion criteria are decided for the study population. These are partially defined by the research question and hypotheses.

\section{Generalisability}

The generalisability of the research findings is dependent on the group that is targeted. If conclusions are to be generalise to the whole country, participants should ideally be drawn from different centres (urban and rural) across the whole geographical area. It must also be considered whether the study population should include patients in hospitals, community clinics and primary care or be from the nation at large. Considerable variation is therefore required in the recruitment strategies used to find participants, and which range from attending ward rounds to knocking on people's front doors.

\section{Representative sample}

Once the study population has been determined, the next step is to consider how to obtain a representative sample of that population. Failure to do so will result in poor generalisability of the results, even for the geographical area under investigation. Specific attention may be required for the adequate recruitment of participants from ethnic minority, non-English speaking, elderly, cognitively impaired or homeless groups. These groups may require different approaches, with the initial emphasis being on building trust and aligning the research goals with those of the minority community
(Levkoff et al, 2000; McNeilly et al, 2000). To achieve this, bilingual or same-culture staff can be hired to target community leaders from religious centres, day centres and action groups. Patients detained in hospital are another important group that is often inadequately represented in research. In some cases, more extreme clinical presentations make it difficult to conduct the lengthy assessments required for research. However, this is not a sufficient reason to exclude all such patients. Thus, careful consideration (and justification) must be given to the process of setting the inclusion and exclusion criteria in research as well as to the subsequent impact that this will have on the recruitment strategies used.

\section{'Normal' control participants}

Some studies require the selection and recruitment of an appropriate comparison group, and 'normal' healthy participants may be selected. A variety of recruitment strategies can be used, including advertising in job-centres and supermarkets. It is often very tempting for the researcher to recruit friends and colleagues and, in some cases, students are given course credits if they 'volunteer' to take part (Lyddy, 2002). Such a situation causes concern as participants should be able to volunteer freely. Furthermore, recruiting university-educated volunteers may not allow for an appropriate comparison with the patient participant group, both in terms of basic demographic factors (e.g. social class, level of education) and core personality traits. Perhaps in an attempt to overcome such difficulties, a Centralised Recruitment Program has been developed in New York. This maintains a pool of healthy participants who are interested in taking part in research (Schechter et al, 1994). Although initially these individuals are not directly recruited to a specific study, a participant self-selection bias is, none the less, still evident.

\section{Response rates}

The response rate is the proportion of those approached who eventually agree to participate in research. It is critically dependent on the selection of the sample of potential participants, the nature of the research and the recruitment strategies utilised. Overall response rates can be enhanced by using a multi-tiered strategy for all initial non-respondents (see 'Assertive tracking' below). Therefore, nonrespondents are approached a second or even a third time, as required, until a response is achieved (even if it is a refusal to participate). However, researchers should note that the specific response rate will usually be highest for the first tier and will reduce by approximately half for each subsequent tier 


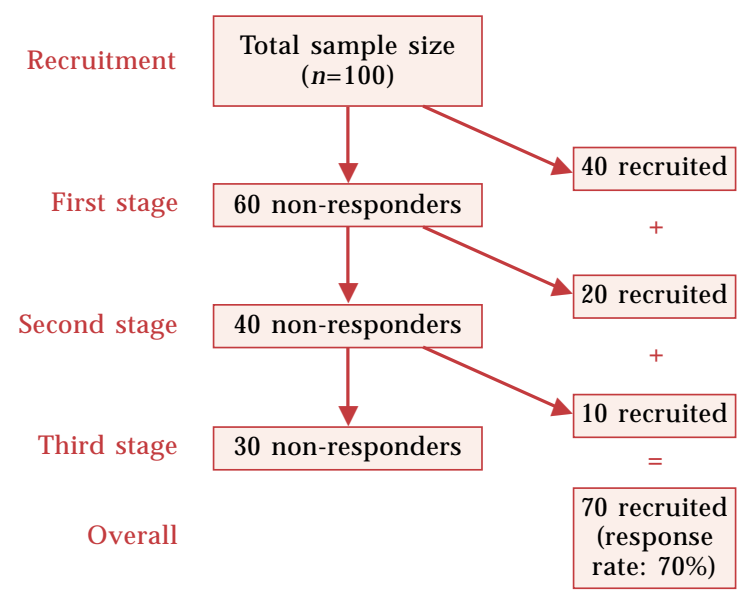

Fig. 2 Response rate decay over three stages.

(Fig. 2). There is a general consensus that response rates of $70 \%$ and above are necessary to ensure that the obtained sample group is sufficiently representative of the target population from which its members are drawn. The assumption here is that the remaining non-respondents are unlikely to have markedly different views and presentations from those that have been successfully recruited into the sample. Low response rates are criticised on the basis of a non-response bias, i.e. the views of the nonresponders may significantly differ from those identified in the recruited group. Attempts to overcome this include demonstrating the lack of significant differences in basic characteristics and demographics between the non-responders and responders.

\section{Working with other clinicians}

Most research studies of human participants involve working with other mental health care professionals. Box 3 summarises the key points of collaboration, which are examined in greater detail below.

\section{Establishing collaboration}

Successful recruitment of participants (in the clinical setting) is critically dependent on initial contacts made with administrative and clinical staff from key recruitment sites. Attention should be paid to factors such as administrative support and the attitude of clinical staff, volume/turnover of patients and stability of the patient population. It is noteworthy that some health professionals advise their administrative staff to discourage calls that include the word 'research'. Clinicians who are supportive of research should be identified at an early stage in the recruitment process. This will save time later on by avoiding unproductive visits to less-thanenthusiastic parties in the future. Attempts at understanding the motivational needs of clinicians can lead to a more accurate perception of how to reach willing collaborators (Young \& Dombrowski, 1989).

It is essential to seek permission at an early stage to carry out a study. Potential collaborators need to know exactly what they will be asked to do, how much time they will be expected to give and what use will be made of the information they provide. Health professionals, administrators, medical records personnel and ward clerks will have to be convinced of the researcher's integrity and the value of the research before they decide whether or not to cooperate (Bell, 1993; Miller et al, 1998). In this regard, it is helpful if the junior researcher has had a few years of clinical experience and if the principal investigator makes a high-profile appearance at the early stages of planning recruitment.

\section{Methods of collaboration}

Pre-study promotion is usually targeted at potential referrers. Various methods are used, including presentations, specially arranged meetings, forums and road shows. During this phase, clear procedures for recruitment should be established between the researcher and the referring clinicians. Mutually agreed procedures should be developed and clearly documented. Eligibility criteria should be clearly stated and recruitment materials (patient information sheets, consent forms) must be provided. For clinical trials, the role of the responsible medical officer must be clarified. Procedures during acute or emergency situations should be outlined and, ideally, rehearsed. Care must be taken not to make excessive demands on the time and effort of the community mental health team (CMHT) (Bell, 1993).

\section{Maintaining collaboration}

During the study, it is important to maintain frequent contact with a nominated member of the referring CMHT, both by telephone and by visits to the refer-

\section{Box 3 Working with colleagues}

Do not underestimate the importance of initial contact with staff from key recruitment sites

Provide adequate information using a variety of methods

Clarify roles, responsibilities and expectations of potential collaborators

Show appreciation for help received

Remember to give feedback 


\section{Box 4 Techniques}

Consider the participant's perspective (costs and benefits)

Stress the relevance of the research

Enhance generic and personal skills and interpersonal communication skills

Establish adequate training and supervision, targets and investigator incentives

Implement assertive tracking procedures using various modes of communication

ring site. The researcher should make an effort to identify convenient opportunities to promote the study and to recruit participants. This can be achieved by volunteering to offer services such as clinical sessions, attending ward rounds and teaching. The question 'What can I do for them [the collaborators] so that they will refer patients to the study?' should constantly engage the researcher. Appreciation should be demonstrated in reciprocation for help given by collaborators. Lastly, regular feedback on results of the investigation and changes in mental state (for individual patients) should be provided to the CMHT as well as information about the overall findings of the research. This aspect is often overlooked and can have profound adverse consequences on future attempts to recruit participants in the local region.

\section{Recruitment techniques}

Techniques to enhance recruitment include addressing the participant's willingness to take part, the investigator's personal performance and utilisation of appropriate methods (Box 4).

\section{The participant's perspective}

Participants take part in research voluntarily, hoping that this will bring them direct therapeutic benefits or will ultimately improve treatment for future patients. Respect for participants (and their relatives and carers) can help to establish trust and rapport (Miller et al, 1998). Few participants will take part in research unless they can identify with and understand its validity and relevance. Consequently, an adequate, clear and concise explanation must be provided (this is also essential when obtaining informed consent).

Presumably, participants conduct a personal cost-benefit analysis when deciding whether or not to participate in research. Attention to these issues may gain the participant's confidence, with resultant successful recruitment (Box 5; Sibbald et al,
1994; Hough et al, 1996; Ribisl et al, 1996; Dowling \& Wiener, 1997; BootsMiller et al, 1998; Lindenberg et al, 2001). Time, travel, financial and inconvenience costs for participants can be minimised if researchers are accommodating and flexible. Financial incentives can be effective but are not significantly motivating for all participants. However, enclosing a gratuity with a postal survey can double the response rate compared with the situation when the incentive is promised contingent on survey completion and return.

\section{The investigator}

Certain personal attributes are an asset to any research investigator. These include being conscientious, having professional integrity and paying meticulous attention to detail. Good interpersonal skills should include the ability to be respectful, tolerant, tactful and approachable and to show a caring and compassionate attitude. Experience in health services and familiarity with the specialist field and the target population are desirable. Appropriate training and regular supervision are also essential prerequisites and these should cover: briefing on the objectives of the project; confidentiality procedures; personal safety; observation and practice sessions under supervision; learning from colleagues who have experience in the same area; and giving attention to the tracking of potential participants. Together with the junior investigator's high visibility at target outreach sites, these factors

Box 5 The participant's perspective - costs and benefits

Costs

Undesired experimental design

Requirement of regular or costly travel to investigation site

Lengthy or frequent appointments required

Tedious, repetitive and lengthy questionnaires

Discontinuation of current treatment

Risk of adverse side-effects from starting new treatment

Risk of being randomised to a placebo group

Benefits

Increased enjoyable personal contact (with services)

Accessibility and affordability of therapy

Altruistic intent

Small financial incentives (to offset costs of time and travel)

Alternative incentives: food vouchers, T-shirts, mugs, book tokens, cards 
will help the investigator to build strong relationships with potential referrers and participants.

Although paying investigators on a piece rate basis may enhance recruitment, not all of them will be motivated by this. The associated personal financial insecurity can lead to rapid staff turnover, which is undesirable in the long run. A fixed salary or even a hybrid approach, using both a fixed salary and incentive bonuses for achieving targets, are preferred alternatives.

\section{Methods}

Methods and tools used to increase awareness of and accessibility to a study during the recruitment phase are shown in Box 6 (Sibbald et al, 1994; Desmond et al, 1995; Coen \& Patrick, 1996; Ribisl et al, 1996; BootsMiller et al, 1998; Fischer et al, 2001). Ideally, the chosen recruitment methods should fit the target population and sample size, although resources and costs may ultimately bias the choice.

Box 6 Methods and tools used to increase study awareness and accessibility

General

A project logo

Business cards

Freephone number and 24-hour answering machine

Websites

Mailshots (mass or targeted)

Fliers/posters in key locations (see below)

General public

Radio

Press releases to local and national newspapers

Key locations - public access buildings, including:

- community centres

- supermarkets

- cinemas

- libraries

- laundrettes

- religious buildings

- employment centres

Clinical groups

Announcements and presentations to potential referrers or participants by:

- arranged meetings

- forums (user-groups)

- road-shows

Key locations - specific buildings, including:

- accommodation facilities

- mental health community facilities

- clinics and hospitals

\section{Assertive tracking}

The first rule is to not give up after the first attempt to recruit a potential participant. Regardless of whether early attempts were initially successful, they should be followed up. Second, many researchers advocate the use of different methods of communication with participants, including letter, telephone, e-mail or face-to-face contact the significance of personal contact should not be underestimated. Using a different method of communication on subsequent attempts to establish contact may yield new success and thus achieve good response rates (Lindenberg et al, 2001; Edwards et al, 2002).

\section{Locator details and collateral contacts}

When a potential participant is first identified, contact should be established wherever possible. Confirm the contact details at each subsequent contact to avoid early attrition before recruitment is completed. Mobile telephone numbers and e-mail addresses may remain unchanged despite many changes of home address, so always ask for these details where appropriate. Also include contact details of collateral agencies (out-patient services, key workers), families and friends. Participants should be asked to sign release of information forms, allowing contact with these agencies and individuals in order to obtain updated details, if required. The participant's right to confidentiality is a key issue here and this principle should not be compromised (Cohen et al, 1993; BootsMiller et al, 1998; Nishimoto, 1998).

\section{Modes of communication}

Letters and e-mails should adhere to the usual rules of written professional correspondence. Some participants (patients and health professionals) respond positively if a letterhead from a medical charity or university is used and personalised with an original signature or a handwritten note. Use of colour (ink, letter paper, enclosed fliers or even envelopes) has been shown to increase response rates. Including a stamped addressed reply envelope and not using a franking machine and computer-generated address labels are also beneficial (Sloan et al, 1997; Edwards et al, 2002). Establishing contact by telephone usually requires several attempts at different times and on different days. Where postal questionnaires have failed, telephone interviews can increase the response rate by a further $30 \%$, although telephone responses are presumably more susceptible to a social desirability response bias and thus may introduce a mode error (Sibbald et al, 1994; Ribisl et al, 1996; BootsMiller et al, 1998; Fischer et al, 2001). 


\section{The researcher's personal perspective}

Recruiting participants can be an emotionally turbulent and maturing experience for a junior researcher. More personal issues are considered below, together with potential solutions.

\section{Team membership}

Recruitment is seldom a solitary exercise. The researcher is usually part of a multi-disciplinary team of researchers headed by a principal investigator, who is generally the grant holder and may be a senior lecturer, professor or consultant. Other members of the team include junior doctors, psychologists, epidemiologists, sociologists and nurses. Differences in expertise, skills, expectations and working styles can affect the individual's role within the team, with varying consequences. However, the team may sometimes consist of only two members: the junior researcher responsible for recruitment and the principal investigator (Young \& Dombrowski, 1989).

\section{Compromised autonomy and emotions}

In a clinical trial, team members responsible for recruiting are often employed after the projected recruitment targets have been decided by the principal investigator or the trial sponsors. This prevents any negotiation by junior researchers. Often, it is not clear from the outset of the study which recruitment strategy is going to work. Together, these issues can leave the junior researcher feeling powerless and uncertain as to how to proceed. In multi-centre studies, where recruitment is competitive, comparison of rates of recruitment creates additional pressure on the team to perform better. When recruitment rates fall behind target, junior researchers should beware that some principal investigators use negative pressure to 'enhance' motivation.

More desirable motivating factors include the opportunity to acquire research skills and experience, to learn how to work in a team and promises of attendance at conferences and of having work published at the end of the study. These should be borne in mind not only at the beginning of the study but also during the difficult times when enthusiasm wanes. However, as time progresses, the initial enthusiasm for recruitment gradually fades. In the face of the harsh realities of research, such as slow recruitment, negative emotions often become dominant. Researchers may experience feelings of despair, self-blame, guilt, a sense of failure, worthlessness, loneliness, frustration and subclinical depression; sometimes even paranoia sets in

\section{Solutions}

It is important to maintain a sense of proportion and objectivity in the face of adversity. This can be achieved by talking to supportive members of the research team as well as to senior colleagues or peers who can give objective advice based on their own research experience. During these sessions, it is important to identify your strengths and weaknesses. Failure to do this can lead to personal distress and have unknown effects on the validity of the research data. It can also contribute to increased costs resulting from burnout (Cohen et al, 1993). You need to be flexible, proactive and creative in finding solutions to problems that had not been anticipated. For example, when working in a catchment area which is overresearched, you should be aware of competition from other researchers interested in the same participant group. Such colleagues may be hostile and should be dealt with diplomatically. The usual solution is to move to another hospital or catchment area where there is less competition and where staff are more receptive to research. Learning how to recruit participants is a significant milestone in anyone's career. If achieved, self-knowledge, enhanced skills and the ability to embrace uncertainty with courage are more than worthy rewards.

\section{Summary}

There are many aspects that the diligent researcher should consider when attempting to maximise participant recruitment and avoid subsequent attrition. These include the following.

\section{Early considerations}

Several aspects of the design and method (e.g. participant exclusion criteria and the required duration of active participation) are necessarily decided at an early stage in the research process. Remember that such decisions will affect actual participant recruitment.

\section{The participant's perspective}

Try to be amenable and accommodating with potential participants. Do not expect them to travel long distances to see you.

\section{Close collaboration with colleagues}

Optimise your working relationship with colleagues by regular face-to-face contact. Be approachable, easily contactable and amenable. 


\section{Generic skills}

Do not underestimate the importance of personal generic skills. Assertive tracking of potential participants requires thorough, methodical and timely organisation. Similarly, it is essential to have a range of good interpersonal and communication skills, using a variety of different methods (e.g. the written word, the telephone and visual presentations).

\section{Feedback}

Lastly, we strongly advocate that researchers should always attempt to present their findings to all colleagues involved in the recruitment phase as well as to the participants. This aspect is often overlooked and can have profound adverse consequences on future attempts to recruit participants in the region.

\section{References}

Armstrong, B. K., White, E. \& Saracci, R. (1992) Principles of Exposure Measurement in Epidemiology. Oxford: Oxford University Press.

Ashery, R. S. \& McAuliffe, W. E. (1992) Implementation issues and techniques in randomised controlled trials of outpatient psychosocial treatments for drug abusers. Recruitment of subjects. American Journal of Drug and Alcohol Abuse, 18, 305-329.

Beauchamp, T. L., Cook, R. R., Fayerweather, W. E., et al (1991) Ethical guidelines for epidemiologists. Journal of Clinical Epidemiology, 44 (suppl. 1), 151S-169S.

Bell, J. (1993) Doing your Research Project. A Guide to Firsttime Researchers in Education and Social Sciences (2nd edn). Buckingham: Open University Press.

BootsMiller, B. J., Ribisl, K. M., Mowbray, C. T., et al (1998) Methods of ensuring high follow-up rates: lessons from a longitudinal study of dual diagnosed participants. Substance Use and Misuse, 33, 2665-2685.

Carpenter, W. T. Jr (1999) The challenge to psychiatry as society's agent for mental illness treatment and research. American Journal of Psychiatry, 156, 1307-1310.

Coen, A. S. \& Patrick, D. C. (1996) Minimizing attrition in longitudinal studies of special populations: an integrated management approach. Evaluation and Program Planning, 19, 309-319

Cohen, E. H., Mowbray, C. T., Bybee, D., et al (1993) Tracking and follow-up methods for research on homelessness. Evaluation Review, 17, 331-352.

Desmond, D., Maddux, J. F., Johnson, T. H., et al (1995) Obtaining follow-up interviews for treatment evalu-ation. Journal of Substance Abuse Treatment, 12, 95102.

Dowling, G. A. \& Wiener, C. L. (1997) Roadblocks encountered in recruiting patients for a study of sleep disruption in Alzheimer's disease. Journal of Nursing Scholarship, 29, 5964.

Edwards, P., Roberts, I., Clarke, M., et al (2002) Increasing response rates to postal questionnaires. Systematic review. $B M J, 324,1183-1185$.

Fischer, E. H., Dornelas, E. A. \& Goethe, J. W. (2001) Characteristics of people lost to attrition in psychiatric follow-up studies. Journal of Nervous and Mental Disease, 189, 49-55.
Hough, R. L., Tarke, H., Renker, V., et al (1996) Recruitment and retention of homeless mentally ill participants in research. Journal of Consulting and Clinical Psychology, 64, 881-891.

Hulley, S. B., Cimmings, S. R., Browner, W. S., et al (2001) Designing Clinical Research. An Epidemiologic Approach (2nd edn). London: Lippincott Williams and Wilkins.

Keith, S. J. (2001) Evaluating characteristics of patient selection and dropout rates. Journal of Clinical Psychiatry, 62 (suppl. 9), 11-14.

Levkoff, S. E., Levy, B. R. \& Weitzman, P. F. (2000) The matching model of recruitment. Journal of Mental Health and Aging, 6, 29-38.

Lindenberg, C. S., Solorzano, R. M., Vilaro, F. M., et al (2001) Challenges and strategies for conducting intervention research with culturally diverse populations. Journal of Transcultural Nursing, 12, 132-139.

Lyddy, F. (2002) Psychology study - any volunteers? Psychologist, 15, 140.

McNeilly, M., Musick, M., Efland, J. R., et al (2000) Minority populations and psychophysiologic research. Challenges in trust building and recruitment. Journal of Mental Health and Aging, 6, 91-102.

Michels, R. (1999) Are research ethics bad for our mental health? New England Journal of Medicine, 340, 1427-1430.

Miller, F. G., Rosenstein, D. L. \& DeRenzo, E. G. (1998) Professional integrity in clinical research. JAMA, 280, 1449-1454.

Nishimoto, R. H. (1998) Who drops out of drug-user treatment research on women? Substance Use and Misuse, 33, 1291-1313.

Polgar, S. \& Thomas, S. A. (2000) Introduction to Research in the Health Sciences (4th edn). Edinburgh: Churchill Livingstone.

Ribisl, K. M., Walton, M. A., Mowbray, C. T., et al (1996) Minimizing participant attrition in panel studies through the use of effective retention and tracking strategies: reviews and recommendations. Evaluation and Program Planning, 19, 1-25.

Schechter, D., Strasser, T., Santangelo, C., et al (1994) Normal control subjects are hard to find: a model for centralized recruitment. Psychiatry Research, 53, 301-311.

Sibbald, B., Addington-Hall, J., Brenneman, D., et al (1994) Telephone versus postal surveys of general practitioners: methodological considerations. British Journal of General Practice, 44, 297-300.

Sloan, M., Kreiger, N. \& James, B. (1997) Improving response rates among doctors: randomised trial. $B M J, 315,1136$.

Smith, M. A. \& Senior, C. (2001) The Internet and clinical psychology. A general review of the implications. Clinical Psychology Review, 21, 129-136.

Streiner, D. L. (1990) Sample size and power in psychiatry. Canadian Journal of Psychiatry, 35, 616-620.

Woods, S. J., Ziedonis, D. M., Sernyak, M. K., et al (2000) Characteristics of participants and non-participants in medication trials for treatment of schizophrenia. Psychiatric Services, 51, 79-84.

Young, C. L. \& Dombrowski, M. (1989) Psychosocial influences on research subject recruitment, enrolment and retention. Social Work in Health Care, 14, 43-57.

\section{Multiple choice questions}

1 Recruitment strategies:

a aim to enlist a representative sample of the target population for a research study

$\mathrm{b}$ involve establishing communication between recruiting staff and potential participants

c are seldom used in psychiatric research

d aim to overcome selection bias

e are adequately reported in psychiatric research literature. 
2 To enhance recruitment rates, the assessment should ideally be:

a repetitive

b relevant

c complicated

d lengthy

e valid.

3 Participants are more likely to participate in research as a result of:

a inconvenience

b boredom

c personal contact

d offered incentive

e altruistic intent.

4 For a successful recruitment strategy, recruiting staff should:

a have good interpersonal skills

b work on a voluntary basis

c be replaced every 2 months

d use only one method of communication

e receive appropriate training and regular supervision.
5 The following are associated with increasing recruitment rates for postal questionnaires:

a use of colour in correspondence

b computer-generated subject address labels

c providing a stamped addressed return envelope

d use of a franking machine

e assertive tracking.

\section{MCQ answers}

\begin{tabular}{|c|c|c|c|}
\hline 1 & 2 & 3 & 4 \\
\hline a $\mathrm{T}$ & a F & a F & a $\mathrm{T}$ \\
\hline $\mathrm{T}$ & b $\mathrm{T}$ & b F & b F \\
\hline $\mathrm{F}$ & c F & c $\mathrm{T}$ & c F \\
\hline $\mathrm{T}$ & d F & $\mathrm{d} T$ & d F \\
\hline $\mathrm{F}$ & e $\mathrm{T}$ & e $\mathrm{T}$ & e $\mathrm{T}$ \\
\hline
\end{tabular}

Gaskell Books • College Journals • Preview Online Versions • Gaskell Books • College Journals

\title{
The Royal College of Psychiatrists/Gaskell
}

\author{
will be exhibiting \\ at the
}

American Psychiatric Association Annual Meeting 2003

San Francisco 17-22 May 2003

\section{Come and see us in the Book Fair at Booth 1322}

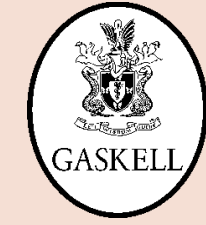

(We are sharing a stand with BMJ Publishing Group) 